\title{
Elliptic Genera and Quantum Field Theory
}

\author{
Edward Witten * \\ Joseph Henry Laboratories, Princeton University, Princeton, NJ 08544, USA
}

\begin{abstract}
It is shown that in elliptic cohomology - as recently formulated in the mathematical literature - the supercharge of the supersymmetric nonlinear sigma model plays a role similar to the role of the Dirac operator in $K$-theory. This leads to several insights concerning both elliptic cohomology and string theory. Some of the relevant calculations have been done previously by Schellekens and Warner in a different context.
\end{abstract}

If $M$ is a spin manifold of dimension $n$, we can consider the Dirac operator $i \not D$, acting on a field $\psi_{\alpha}$ which is a section of the spinor bundle $S$. More generally, if $R$ is any representation of the structure group $\operatorname{Spin}(n)$ of the tangent bundle, we can consider the Dirac operator acting on a field $\psi_{\alpha i}, \alpha$, and $i$ being respectively a spinor index and an index labeling the representation $R$; in mathematical terms, $\psi$ is a section of $S \otimes T_{R}, T_{R}$ being the $\operatorname{Spin}(n)$ bundle associated with the representation $R$ of $\operatorname{Spin}(n)$.

In [1], an infinite series of representations $R_{i}, i=0,1,2, \ldots$ was singled out. The first few were

$$
\begin{aligned}
& R_{0}=1, \\
& R_{1}=T, \\
& R_{2}=\Lambda^{2} T \oplus T, \\
& R_{3}=\Lambda^{3} T \oplus(T \otimes T) \oplus T .
\end{aligned}
$$

Here 1 is the trivial representation, $T$ is the fundamental (vector) representation of $S O(N)$, and $A^{k}$ denotes the $k^{\text {th }}$ antisymmetric tensor product. The special role of these operators was as follows. Let $M$ be a spin manifold with a compact symmetry group $G$. It is sufficient in what follows to consider an $S^{1}$ [i.e., $\left.U(1)\right]$ subgroup of $G$. Let $K$ be the generator of this $S^{1}$ action. Assuming that the symmetry generated by $K$ lifts to the spinor bundle, $K$ commutes with the Dirac operator $i D D$ (or a

\footnotetext{
* Supported in part by NSF grants PHY 80-19754 and PHY 86-16129
} 
generalized Dirac operator acting on sections of $S \otimes T_{R}$ ), so it makes sense to consider the index of the Dirac operator acting on states $\psi$ with

$$
K \psi=k \psi
$$

for fixed $k$. (As $K$ generates an $S^{1}$ action, the eigenvalues $k$ are integers or halfintegers, depending on whether the $S^{1}$ action is "even" or "odd", i.e. depending on whether $e^{2 \pi i K}=+1$ or $e^{2 \pi i K}=-1$.) We will denote the index of the operator $i \not D$ in the subspace of states obeying (2) as $c_{k}$. The function $\sum c_{k} e^{i k \theta}$ is called the character-valued index of the Dirac operator.

For the ordinary Dirac operator, i.e. for $R_{0}=1$, Atiyah and Hirzebruch proved that $c_{k}=0$ for all $k$ [2]. The proof was a simple application of the fixed point form of the index theorem [3]. In [4], it was conjectured for the Rarita-Schwinger case, $R_{1}=T$, that the character-valued index is a constant, i.e. that the $c_{k}$ are all zero for $k \neq 0$. (This was proved in [4] for homogeneous spaces. The restriction to $k \neq 0$ is necessary, since for $H P^{2}$ one has $c_{0}=-1$. For odd actions, $c_{0}=0$ and the restriction on $k$ is unnecessary.) Trying to prove this conjecture by methods of equivariant $S^{1}$ spin bordism, some of whose properties were established in [5], Landweber and Stong established in [1] that $\sum c_{k}=0$ if the $S^{1}$ action is odd and semi-free (for the action to be semi-free means that every $x \in M$ is left fixed by the identity in $S^{1}$ only or by the whole group), and moreover that for semi-free $S^{1}$ actions on spin manifolds the corresponding statement is valid not just for the Rarita-Schwinger operator but for the whole infinite series of operators indicated in (1).

Subsequent work $[6,7,15]$ showed, roughly, that it is possible to use elliptic modular forms to write a generating functional for this infinite series of operators. To be precise, the modular forms in question were modular forms for the congruence subgroup $\Gamma_{0}(2)$ of $S L(2, Z)$, which can be viewed as the subgroup that leaves fixed one of the three non-trivial spin structures on an elliptic curve. Ochanine introduced [6] the notion of an "elliptic genus" and synthesized the above statements in the following conjecture: for any elliptic genus and any spin manifold $M$ with $S^{1}$ action, the character valued elliptic genus is a constant. I will not here give Ochanine's definition of an elliptic genus, since a notion adequate for our purposes will appear presently. Progress toward proving this conjecture was described in [8]; in particular, it was shown that $c_{k}=0$ for odd semi-free actions.

In recent work, Landweber et al. have interpreted the elliptic genus as a natural invariant in a generalized cohomology theory, "elliptic cohomology" [9]. Roughly speaking, elliptic cohomology is proposed as a generalization of $K$-theory, the elliptic genus being related to elliptic cohomology the way the Dirac index is related to $K$-theory. It is then natural to ask whether there is an operator analogous to the Dirac operator - whose index gives the elliptic genus. The purpose of the present paper is to argue that the operator in question is the supercharge of the supersymmetric nonlinear sigma model, and to indicate some of the implications of this for topology and string theory.

Apart from the facts cited above, another clue to the connection between elliptic cohomology and string theory appears in the work of Hopkins, Kuhn, and Ravenel [10], who computed in elliptic cohomology the Euler characteristic of a quotient $M / G$ and obtained formulas similar to those that have appeared in 
orbifold calculations in string theory [11]. Some of the key formulas below have been first written by Schellekens and Warner [12] in their work on anomaly cancellation. They interpreted equations such as (10) below as a generating functional of anomalies.

The present paper is mostly explained in "physical" terms. A mathematical account will be presented elsewhere, with an interpretation of the key formulas in terms of a fixed point theorem for the natural circle action on a Dirac-like operator in free loop space, along the lines of [13].

Looking at the representations in (1), a string theorist may well note that the $k^{\text {th }}$ representation in (1) coincides with what appears at the $k^{\text {th }}$ mass level of the NeveuSchwarz sector of open superstrings. Thus, in this sector, the ground state $|\Omega\rangle$ is a $\operatorname{spin}(n) \operatorname{singlet}$. Excitations are created by fermionic creation operators $\psi_{k}^{i}$, $k=-\frac{1}{2},-\frac{3}{2},-\frac{5}{2}, \ldots$ and bosonic creation operators $\alpha_{k}^{i}, k=-1,-2,-3, \ldots$. At the first excited level, we have $\psi_{-1 / 2}^{i}|\Omega\rangle$, transforming as $T$, at the second level we have $\psi_{-1 / 2}^{i} \psi_{-1 / 2}^{j}|\Omega\rangle$ and $\alpha_{-1}^{i}|\Omega\rangle$, transforming as $\Lambda^{2} T \oplus T$; at the third level we have $\psi_{-1 / 2}^{i} \psi_{-1 / 2}^{j} \psi_{-1 / 2}^{k}|\Omega\rangle, \quad \psi_{-1 / 2}^{i} \alpha_{-1}^{j}|\Omega\rangle, \quad$ and $\psi_{-3 / 2}^{i}|\Omega\rangle$, transforming as $\Lambda^{3} T \oplus(T \otimes T) \oplus T$. Assuming that this continues to all orders, we can write down the "partition function" or generating functional for the representations $R_{k}$. It is

$$
\sum q^{k / 2} R_{k}=\bigotimes_{k=\frac{1}{2}, \frac{3}{2}, \frac{5}{2}, \ldots} \Lambda_{q^{k}} T \underset{l=1,2,3, \ldots}{\bigotimes} S_{q^{l}} T .
$$

Here $\Lambda_{t} T$ is an abbreviation for $1+t T+t^{2} \Lambda^{2} T+\ldots$ and $S_{t} T$ is an abbreviation for $1+t T+t^{2} S^{2} T+\ldots$, with $S^{k} T$ being the $k^{\text {th }}$ symmetric tensor power of $T$. In (3), $q$ is a formal variable; one is to expand in powers of $q$, the coefficient of $q^{k / 2}$ being the $k^{\text {th }}$ representation in the series (1). The Dirac index for spinors with values in the graded representation (3) is a power series in $q$ which can be regarded as the universal elliptic genus; a particular elliptic genus can be obtained by setting $q$ to a definite complex value.

Now, we discuss precisely what operator has the elliptic genus for its index. We will consider a closed type II superstring with Ramond boundary conditions for right movers and Neveu-Schwarz boundary conditions for left movers. The right moving Ramond boundary conditions give spinor quantum numbers, and the leftmoving Neveu-Schwarz boundary conditions give the series of operators (1).

Let $Q$ be the supercharge of the right moving degrees of freedom:

$$
Q=\int_{0}^{2 \pi} d \sigma g_{i j}\left(X^{k}(\sigma)\right) \partial_{+} X^{i}(\sigma) \psi_{+}^{j}(\sigma)
$$

Its square is the Hamiltonian $L_{0}$ of the right moving modes. In $(4) g_{i j}$ is the metric of $M$ and $\sigma$ is a standard angular parameter; $X^{k}$ are coordinates of the manifold $M$ and $\psi^{k}$ are their superpartners. Let $(-1)^{F_{R}}$ be the operator that counts rightmoving fermions modulo two. Then

$$
Q(-1)^{F_{R}}+(-1)^{F_{R}} Q=0,
$$

and we are tempted to define the index of $Q$ to be the number of zero eigenvalues of $Q$ with $(-1)^{F_{R}}=+1$ minus the number with $(-1)^{F_{R}}=-1$. The correct notion in this context, though, is a more refined character-valued index. $Q$ commutes with 
the momentum operator

$$
P=\widetilde{L}_{0}-L_{0}
$$

which generates a rotation ( $\delta \sigma=$ const) of the circle $S^{1}$ on which the sigma model is defined. We denote as $b_{\lambda}$ the index of $Q$ restricted to the subspace $H_{\lambda}$ of Hilbert space in which $P=\lambda$. We then define the character-valued index of $Q$ to be

$$
F(q)=\sum_{\lambda} b_{\lambda} q^{\lambda}
$$

What values of $\lambda$ appear as eigenvalues of $P$ ? At first sight one might expect that they are integers, since $P$ generates a rotation of $S^{1}$, and one might expect that $\exp 2 \pi i P=1$. This is wrong for several reasons. First, because of the leftmoving Neveu-Schwarz boundary conditions, a $2 \pi$ rotation of the circle multiplies the quantum state by a factor of $(-1)^{F_{L}}$. Second, looking at things from a path integral point of view, the $2 \pi$ rotation of the circle is an example of a modular transformation $(\tau \rightarrow \tau+1)$. Under this transformation, there is a global anomaly in the fermion determinant, and the quantum wave function acquires a phase $e^{-i \pi d / 8}$ under a $2 \pi$ rotation, $d$ being the dimension of $M$. The correct operator statement is

$$
\exp 2 \pi i P=(-1)^{F_{L}} \exp -i \pi d / 8 \text {. }
$$

The eigenvalues of $P$ are therefore of the form $\lambda=-\frac{d}{16}+\frac{n}{2}$, where $n$ is an integer, even or odd for $(-1)^{F_{L}}=+1$ or $(-1)^{F_{L}}=-1$. The $-d / 16$ is just the ground state energy of the Neveu-Schwarz sector. Since the $\exp (-i \pi d / 8)$ in (7) comes from a topological invariant, a global anomaly in the fermion determinant, quantum corrections in the sigma model do not shift this factor from its free field theory value.

We thus rewrite (7) as

$$
F(q)=q^{-d / 16} \sum_{l \in Z} b_{l} q^{l / 2}
$$

to emphasize that the eigenvalues of $P$ range over a known, discrete set. We now can assert that the $b_{l}$, being integers, must be topological invariants, unchanged under any smooth deformation of the nonlinear sigma model under discussion. In particular, we can go over to a limit in which the radius of $M$ is very large, that is, in which the metric of $M$ is $g=t^{2} g_{(0)}$, where $g_{(0)}$ is some fixed metric and we take $t \rightarrow \infty$. The spectrum of $Q$ can then be worked out in a systematic expansion in powers of $1 / t$. In flat space, states of $L_{0}=0, \widetilde{L}_{0}=P=-\frac{d}{16}+\frac{l}{2}$ have quantum numbers $S \otimes R_{l}$. Therefore, in the leading large $t$ approximation, the $Q$ eigenvalues with small $L_{0}$ and $P=-\frac{d}{16}+\frac{l}{2}$ are just the eigenvalues of the ordinary finite dimensional Dirac operator $i \not D$ acting on sections of $S \otimes R_{l}$. In particular, $b_{l}$ is equal to what we will call index $\left(R_{l}\right)$, the index of the Dirac operator in this representation, and thus

$$
F(q)=q^{--d / 16} \sum_{l=0,1,2, \ldots} \operatorname{index}\left(R_{l}\right) \cdot q^{l / 2} .
$$


Using the characterization (3) of the $R_{k}$ and the ordinary Atiyah-Singer index theorem [14] to determine the integers index $\left(R_{l}\right)$, we have

$$
F(q)=q^{-d / 16}\left(\hat{A}(M) \mathrm{ch} \bigotimes_{k=\frac{1}{2}, \frac{3}{2}, \frac{5}{2}, \ldots} \Lambda_{q^{k}} T \bigotimes_{l=1,2,3, \ldots} S_{q^{l}} T\right)[M] .
$$

While not obviously equivalent to the original definitions of the elliptic genus, (11) can be shown to be equivalent to them [9]. Indeed, one can show from (11) that

$$
F(q)=\left(\frac{\eta\left(-q^{1 / 2}\right)}{\eta(q) \eta(-q)}\right)^{d} \Phi(q),
$$

with $\eta(q)$ the Dedekind eta function, and $\Phi(q)$ a modular form of weight $d / 2$ for the congruence subgroup $\Gamma_{0}(2)$ mentioned earlier. $\Phi(q)$, which according to (11) is determined by characteristic numbers of the manifold $M$, is what has been defined in $[6,9]$ as the elliptic genus. This has been proved explicitly by Landweber [9] and Zagier [15]. The explicit formulas that arise are similar to what we will meet in Eq. (16) below.

However, the simple modular properties of (11) have a natural "physical" explanation. We can write

$$
F(q)=\operatorname{Tr} q^{\bar{L}_{0}} \bar{q}^{L_{0}}(-1)^{F_{R}},
$$

since, in the usual way, states of $L_{0} \neq 0$ will cancel out of the trace. Equation (13) can then be represented by a path integral on an elliptic curve defined by a lattice $(1, \tau)$ in the complex plane $\left(q=e^{2 \pi i \tau}\right)$. The subgroup of $S L(2, Z)$ that leaves fixed the right-moving Ramond and left-moving Neveu-Schwarz boundary conditions is $\Gamma_{0}(2)$. The path integral representation makes it clear that $|F(q)|$ is $\Gamma_{0}(2)$ invariant; the behavior of the phase of $F(q)$ under $\Gamma_{0}(2)$ involves the global anomaly noted earlier. It should be noted that, up to phase, the product $\left(\eta(q) \eta(-q) / \eta\left(-q^{1 / 2}\right)\right)^{d}$ transforms with weight $d / 2$; this is why $\Phi(q)$ has weight $d / 2$.

The ring of modular forms for $\Gamma_{0}(2)$ is isomorphic to $C[\delta, \varepsilon]$, where $\delta$ and $\varepsilon$ are generators of weight two and four, respectively. In [9], it has been found that periodic cohomology theories can be obtained with $Z\left[\frac{1}{2}\right]\left[\delta, \varepsilon, \varepsilon^{-1}\right]$ or $Z\left[\frac{1}{2}\right]\left[\delta, \varepsilon,\left(\delta^{2}-\varepsilon\right)^{-1}\right]$ as coefficient ring.

In fact,

$$
\varepsilon\left(q^{1 / 2}\right)=\left(\frac{\eta(q) \eta(-q)}{\eta\left(-q^{1 / 2}\right)}\right)^{8},
$$

so the quantum field theory is producing $\Phi(q) \varepsilon\left(q^{1 / 2}\right)^{-d / 8}$ for the elliptic genus. This perhaps is a suggestion that it is $\varepsilon$ that should be inverted in defining the cohomology theory.

The remainder of this paper will be devoted to a brief discussion of mathematical and physical applications of the connection between quantum field theory and the elliptic genus.

Considering first mathematical applications, let us examine in the light of quantum field theory the question which motivated the development of elliptic genera and elliptic cohomology. Thus, as in our initial comments, let $M$ be a spin manifold with $S^{1}$ action, generated by $K$. We have then a more refined notion of a character valued index. The supercharge commutes with the $S^{1} \times S^{1}$ action 
generated by $P$ and $K$. Let $b_{l, k}$ be the index of the supercharge $Q$ for states of $P=-\frac{d}{16}+\frac{l}{2}, K=k$. Thus,

$$
\operatorname{Tr} q^{\bar{L}_{0}} \bar{q}^{L_{0}} e^{i \theta K}(-1)^{F_{R}}=q^{-d / 16} \sum_{l, k} b_{l, k} q^{l / 2} e^{i k \theta} .
$$

We know that $b_{l, k}=0$ for $l \ll 0$ since the Hamiltonian of the quantum field theory is bounded below. Actually since the $b_{l, k}$ are topological invariants, they vanish if $-\frac{d}{16}+\frac{l}{2}$ is less than the ground state energy in the large radius limit. Consequently

$$
b_{l, k}=0 \quad \text { if } \quad l<0 .
$$

We would like to prove that $b_{l, k}=0$ if $k \neq 0$.

To do so, we generalize the problem and consider a one parameter family of theories with "twisted boundary conditions." Without spoiling supersymmetry, we can consider a one parameter family of nonlinear sigma models, with boundary conditions

$$
\begin{aligned}
& X^{i}(\sigma+2 \pi)=e^{i \alpha K} X^{i}(\sigma), \\
& \psi_{R}^{i}(\sigma+2 \pi)=e^{i \alpha K} \psi_{R}^{i}(\sigma), \\
& \psi_{L}^{i}(\sigma+2 \pi)=-e^{i \alpha K} \psi_{L}^{i}(\sigma) .
\end{aligned}
$$

Mathematically, this means that (for instance) $\sigma \rightarrow X(\sigma)$ no longer defines a map $S^{1} \rightarrow M$, but a section of a twisted $M$ bundle over $S^{1}$.

With the boundary conditions (17), the operator relation (8) is modified to

$$
\exp 2 \pi i P=(-1)^{F_{L}} \exp i \alpha K \exp -\frac{i \pi d}{8},
$$

since now a $2 \pi$ rotation of $S^{1}$ brings about an extra transformation by $e^{i \alpha K}$. This shifts the allowed eigenvalues of $P$, which are now of the form

$$
\lambda=-\frac{d}{16}+\frac{n}{2}+\frac{\alpha k}{2 \pi}
$$

where $k$ is the eigenvalue of $K$ and $n$ is as before. At general $\alpha,(15)$ is thus replaced by

$$
\operatorname{Tr} q^{\bar{L}_{0}} \bar{q}^{L_{0}} e^{i \theta K}(-1)^{F_{R}}=q^{-d / 16} \sum_{n, k} b_{n, k}(\alpha) q^{n / 2+\alpha k / 2 \pi} e^{i k \theta}
$$

By continuity, the integers $b_{n, k}(\alpha)$ are independent of $\alpha$. Setting $\alpha=2 \pi$, we learn from (20) that

$$
\operatorname{Tr} q^{\bar{L}_{0}} \bar{q}^{L_{0}}(-1)^{F_{R}}=q^{-d / 16} \sum_{n, k} b_{n, k} q^{n / 2+k} e^{i k \theta} .
$$

But $\alpha=2 \pi$ is equivalent to $\alpha=0$. So, comparing (21) to (15), we find

$$
b_{n, k}=b_{n+2 k . k} \text {. }
$$


Iterating the identity (22) $t$ times, for any integer $t$, we learn

$$
b_{n, k}=b_{n+2 t k, k} \text {. }
$$

Together with (16), this gives us the desired result

$$
b_{n, k}=0 \text { for } k \neq 0 .
$$

To make this argument into a rigorous proof, a cut-off version of the nonlinear sigma model might suffice. The cutoff of [16] or [17] might be suitable. The properties of quantum field theory used in the argument are relatively modest and certainly do not include relativistic invariance.

Continuing with our discussion of mathematical applications of the connection between quantum field theory and the elliptic genus, we note that alternative nonlinear sigma models will give rise to alternative elliptic genera. For example, consider an $N=\frac{1}{2}$ supersymmetric nonlinear sigma model with right-moving fermions only, coupled to the spin connection of the manifold $M$. Adopting periodic boundary conditions for the right-moving fermions (in both $\sigma$ and $\tau$ directions), one sees that

$$
G(q)=\operatorname{Tr} q^{\bar{L}_{0}} \bar{q}^{L_{0}}(-1)^{F_{R}}
$$

will transform simply under the full modular group $S L(2, Z)[S L(2, Z)$ invariance is violated only by the global anomaly], since the fermion boundary conditions are $S L(2, Z)$ invariant. The formula analogous to (11) is

$$
G(q)=q^{-d / 24}\left(\hat{A}(M) \operatorname{ch} \underset{l=1,2,3, \ldots}{\otimes} S_{q^{l}} T\right)[M] .
$$

This will have a simple transformation law under $S L(2, Z)$ provided $M$ is a spin manifold with $p_{1}(M)=0$, so that the nonlinear sigma model is free of anomalies. In fact, under this condition

$$
G(q)=\frac{\Phi(q)}{\eta(q)^{d}}
$$

where $\Phi(q)$ is a modular form of $S L(2, Z)$ of weight $d / 2$. The latter statement is closely related to observations by Schellekens and Warner [12] concerning anomalies. They and independently Zagier [15] obtained the formula

$$
\Phi(q)=\exp \left(\sum_{k=1}^{\infty} \frac{2}{(2 k) !} G_{2 k}(q) \sum_{i} u_{i}^{2 k}\right),
$$

where the $u_{i}$ are formal eigenvalues of the curvature two form, and

$$
G_{2 k}=-\frac{B_{2 k}}{4 k}+\sum_{n} \sigma_{2 k-1}(n) q^{n}
$$

is the Eisenstein series of weight $2 k$. The $G_{2 k}$ are modular forms (of weight $2 k$ ) for $k>1 . G_{2}$ will not appear in $(28)$, so that $\Phi(q)$ will be a modular form, precisely if $\sum_{i} u_{i}^{2}=0$, that is if $p_{1}(M)=0$. [The nonlinear sigma model under discussion only makes sense if in addition $M$ is a spin manifold; this is not needed in order for $\Phi(q)$ 
to be a modular form, but it is needed in order for $G(q)$ in (27) to be the charactervalued index of an operator, and so to have integral $q$-expansion.]

Finally, we can consider an $N=\frac{1}{2}$ supersymmetric nonlinear sigma model with additional left-moving degrees of freedom. This is the case most closely related to heterotic strings. If $M$ is endowed with an $N$ dimensional vector bundle $V$ with structure group $S O(N)$, and we introduce $N$ left-moving fermions coupled to $V$, with (say) Neveu-Schwarz boundary conditions, then (11) is replaced by

$$
H(q)=q^{-d / 24-N / 48}\left(\hat{A}(M) \mathrm{ch} \bigotimes_{k=\frac{1}{2}, \frac{3}{2}, \frac{5}{2}, \ldots} \Lambda_{q^{k}} V{\underset{l=1.2 .3 \ldots}{q^{l}}}_{\bigotimes} S T\right)[M],
$$

which might be interpreted as the elliptic index of a vector bundle. If one adopts Ramond boundary conditions for left-moving fermions, (30) is replaced by

$$
\tilde{H}(q)=q^{-\frac{(d-N)}{24}}\left(\hat{A}(M) \operatorname{ch}\left[\left(\Delta_{+}(V)-\Delta_{-}(V)\right) \underset{l=1,2,3 \ldots}{\bigotimes} \Lambda_{-q^{l}} V S_{q^{l}} T\right]\right)[M],
$$

where $\Delta_{+}(V)$ and $\Delta_{-}(V)$ are the positive and negative spinor bundles associated with $V$. The conditions for the quantum field theories that give (30) and (31) to make sense are $w_{2}(V)=w_{2}(T)=0, p_{1}(V)=p_{1}(T)$. [In the case of (31), the former condition can be relaxed to $w_{2}(V)=w_{2}(T)$.] Under these conditions, (30) and (31) lead to modular forms of level two and level one, respectively. The genus (31) is unstable, in contrast to the others considered earlier. It is roughly a generalization to elliptic cohomology of the Euler characteristic of a vector bundle.

If $M$ admits an $S^{1}$ action, one may wonder whether the genera (26), (30), (31) are governed by theorems analogous to the theorem governing the original elliptic genus (11) whose proof we sketched earlier. [In the case of (30) and (31) it is necessary to assume that the $S^{1}$ action lifts to an action on $V$.] Such theorems indeed exist, but the situation is subtler than that surrounding (11) because of the possibility of anomalies in the $S^{1}$ action. To avoid such anomalies, all statements about $p_{1}$ must be replaced by equivariant statements. If $B S^{1}$ is the classifying space of the group $S^{1}$, and $M$ is a manifold with $S^{1}$ action, there is by definition of $B S^{1}$ a corresponding fiber bundle $X$ over $B S^{1}$ with fiber $M . T$ and $V$ are then the restrictions to $M$ of bundles $T^{*}$ and $V^{*}$ over $X$. One says that $p_{1}(T)=p_{1}(V)$ equivariantly if $p_{1}\left(T^{*}\right)=p_{1}\left(V^{*}\right)$. This is the condition for the $S^{1}$ action on $M$ to be anomaly free in the nonlinear sigma model. Under this restriction, the genera (30) and (31) are governed by the same statement as (11): the $S^{1}$ character-valued generalizations of these genera are constants. A subtler theorem arises if $p_{1}\left(T^{*}\right)$ $-p_{1}\left(V^{*}\right)$ is not zero but is the pullback to $X$ of a cohomology class on $B S^{1}$. In this case, the anomaly in the $S^{1}$ action is purely a $c$-number, and the $S^{1}$ charactervalued generalizations of (26), (30), and (31) are not constants but transform as Jacobi forms [18]. I will discuss these matters elsewhere.

Finally, we will devote the rest of this paper to physical considerations concerning elliptic cohomology. If one asks in field theory whether a space-time manifold $M$ is "the same" as a space-time manifold $M^{\prime}$, the answer is that this is so if and only if $M$ and $M^{\prime}$ are isomorphic topologically. Indeed, topology is by definition that which cannot change continuously in field theory.

The answer to the analogous question in string theory is much less obvious. Even if $M$ and $M^{\prime}$ are distinct in the sense of field theory, they might be isomorphic 
in string theory. One way to pose this question involves considering nonlinear sigma models. For simplicity, consider first bosonic sigma models. The simplest class of sigma models on a manifold $M$ depends on the choice of a metric $g_{i j}$ on $M$. The action is

$$
I=\frac{1}{2} \int_{\Sigma} d^{2} \sigma \partial_{\alpha} X^{i} \partial^{\alpha} X^{j} g_{i j}\left(X^{k}(\sigma)\right)
$$

with $\Sigma$ being a Riemann surface. More generally, giving expectation values to vertex operators of massive fields, we consider

$$
I^{\prime}=\frac{1}{2} \int_{\Sigma} d^{2} \sigma\left(\partial_{\alpha} X^{i} \partial^{\alpha} X^{j} g_{i j}\left(X^{k}(\sigma)\right)+\partial_{\alpha} X^{i} \partial^{\alpha} X^{j} \partial_{\beta} X^{l} \partial^{\beta} X^{m} u_{i j l m}\left(X^{k}(\sigma)\right)+\ldots\right)
$$

a sigma model with arbitrary couplings.

Now, in the generality of (33), it is not at all obvious that topologically distinct manifolds give rise to distinct sigma models. Given a simple sigma model (32) on some manifold $M$, perhaps with some cunning choice of couplings it is equivalent to a complicated quantum field theory (33) on the manifold $M^{\prime}$. In this case, the two manifolds $M$ and $M^{\prime}$ would really be equivalent in string theory; by suitable adjustment of couplings in (33), one could interpolate smoothly from a simple quantum field theory on $M$ to a simple one on $M^{\prime}$. The case of a group manifold, which under appropriate conditions gives the same sigma model as a flat torus, is an example of this [19].

The issue is thus to distinguish topologically not just between manifolds $M$ and $M^{\prime}$ but between quantum field theories in which the target spaces are $M$ and $M^{\prime}$. To gain some insight into this question, we must find topological invariants associated with quantum field theories. For bosonic sigma models, it is not clear how to do this. For supersymmetric sigma models, though, the situation is different. Given a sigma model with $(1,1)$ supersymmetry, that is, with both leftand right-moving $N=1$ supersymmetry, we are free to choose fermion boundary conditions for both left- and right-moving modes. We have seen that with leftmoving Neveu-Schwarz boundary conditions and right-moving Ramond boundary, we can extract a topological invariant, the elliptic genus. If the elliptic genera of two manifolds $M$ and $M^{\prime}$ are not equal, then the $(1,1)$ supersymmetric sigma models based on $M$ and $M^{\prime}$ are topologically distinct. Likewise, by choosing Ramond boundary conditions for both left-and right-movers, we can compute the ordinary supersymmetric index $\operatorname{Tr}(-1)^{F}$, which equals the Euler characteristic of $M$. We conclude that the sigma models based on $M$ and $M^{\prime}$ are topologically distinct if $M$ and $M^{\prime}$ have distinct Euler characteristics. The appropriate elliptic genus, but not the Euler characteristic, is likewise an invariant for sigma models with right-moving supersymmetry only.

These are some of the simplest topological invariants of nonlinear sigma models, but there are certainly others. For instance, apart from the ordinary Dirac index, one has in conventional Riemannian geometry mod 2 counterparts of the Dirac index which appear in $8 k+1$ and $8 k+2$ dimensions. In $8 k+1$ dimensions, the mod 2 index arises because the spinor bundle is real, and the Dirac operator $i \not D$ is imaginary and hermitian. It follows that if $\lambda$ is an eigenvalue of $i \not D$, so is $-\lambda$, with the same multiplicity. From this one at once deduces that the number of zero eigenvalues of $i \not D$ is a topological invariant modulo 2 [14]. 
An analogous argument is possible for supersymmetric nonlinear sigma models. The role of complex conjugation is played by the CPT operation $\Theta$, an anti-unitary operation which we can take to obey $\Theta^{2}=1$. Let $Q$ be the rightmoving supercharge. The argument will depend on the nature of the left-moving degrees of freedom in a way that will become clear.

Assuming that there is only one right-moving supercharge, $\Theta Q \Theta$ must be a multiple of $Q$,

$$
\Theta Q \Theta= \pm Q
$$

However, the sign \pm 1 on the right-hand side of (34) depends on the dimension $d$ of the target manifold $M$ and the choice of left-moving degrees of freedom. The sign can be determined as follows. Since the possible values are discrete, the sign is a topological invariant and can be computed by going over to the large radius, field theory limit. In that limit, $Q$ reduces to $i \not D$ acting on spinors with values in some vector bundle $V$ which depends on the choice of left-moving degrees of freedom. ( $V$ is simply determined by the ground state in the left-moving Hilbert space.) If, for instance, left-moving spinors are absent, or obey Neveu-Schwarz boundary conditions, or describe $E_{8}$ current algebra, $V$ is trivial. In that case, the conventional analysis shows that in $8 k+1$ dimensions, the sign in (34) is -1 .

Just as in the classical situation, the relation $\Theta Q \Theta=-Q$ for appropriate nonlinear sigma models in $8 k+1$ dimensions means that the number of zero eigenvalues of $Q$ is a topological invariant modulo 2 . Actually, we can do better. As $\Theta$ and $Q$ commute with the momentum operator $P$, one obtains a $\bmod 2$ invariant for each eigenvalue of $P$. It is interesting to speculate that these might sum up to some kind of mod 2 modular form.

Similar remarks could be made about $\bmod 2$ invariants for sigma models in $8 k+2$ dimensions, but I will forgo a discussion of this subject here. Further remarks about the role of the world-sheet CPT operator in string theory can be found in the appendix.

A further comment about topological invariants of sigma models is in order. Even if two manifolds $M$ and $M^{\prime}$ have different dimension, the corresponding sigma models might be isomorphic. For instance, in the case of bosonic sigma models, a model defined on a group manifold can be equivalent to a model defined on a flat manifold of dimension much lower. However, we will now argue that for supersymmetric sigma models there are strong restrictions on this phenomenon.

We have already seen in the discussion surrounding Eq. (34) how to extract a dimension dependent invariant from a supersymmetric sigma model. One could try to proceed in a similar vein and find the whole mod 8 periodicity of the Clifford algebra as an invariant of sigma models. I will instead use another, simpler argument to this effect.

Consider first sigma models with $(1,1)$ supersymmetry. Pick Ramond boundary conditions for right-movers and Neveu-Schwarz boundary conditions for leftmovers. Then, as we have already discussed, in connection with Eq. (8), the global anomaly

$$
\exp 2 \pi i P=(-1)^{F_{L}} \exp (-i \pi d / 8)
$$

is a topological invariant. In particular, the expression $(-1)^{F_{L}} \exp (-i \pi d / 8)$ is an invariant property of a nonlinear sigma model, independent of a particular 
description of the model in terms of a manifold $M$. At first sight, this might seem to show that the dimension $d$ of $M$ is an invariant $\bmod 16$. Actually, the overall sign of the operator $(-1)^{F_{L}}$ is ill-defined [this operator is defined abstractly by saying that it anti-commutes with left-moving fermions, a property that defines $(-1)^{F_{L}}$ only up to sign], so $d$ is really only an invariant $\bmod 8$.

Now consider a sigma model with $(0,1)$ supersymmetry. Pick Ramond boundary conditions for right-movers, and assume that the left-moving internal degrees of freedom are a modular invariant combination, for instance trivial. Then (35) is replaced by $\exp 2 \pi i P=\exp -i \pi d / 12$. This means that for such sigma models, $d$ is an invariant modulo 24 .

The above comments on topological invariants of nonlinear sigma models are certainly far from complete. The hope should be that a proper variant of elliptic cohomology will emerge as the correct framework for this discussion, unifying the above observations and pointing the way to new and perhaps less obvious invariants.

In conclusion, the supersymmetric nonlinear sigma model plays in elliptic cohomology a role similar to the role of the Dirac operator in $K$-theory. Elliptic cohomology is thus likely to enter in illuminating the mysterious generalization of ordinary geometry and topology afforded by string theory. A properly developed theory of elliptic cohomology is likely to shed some light on what string theory really means.

\section{Appendix. CPT Conservation on the World Sheet and in Space-Time}

In this appendix I will briefly describe another role of the world sheet CPT operator, beyond that described in the text.

CPT conservation is well known to be a general property of quantum field theory. String theory generalizes quantum field theory in a still largely mysterious way. It is incumbent upon us to ask whether CPT is conserved in string theory.

We certainly do not understand string theory well enough to prove a general CPT theorem in string theory. However, we can ask whether CPT conservation holds in the framework of the calculations that we are currently able to perform.

Nonlinear sigma models offer a general framework for discussing effective four dimensional physics. Compactification on $M^{4} \times K$ can be described by a suitable sigma model. Why should CPT be valid in this framework?

A typical consequence of CPT is the equality of particle and anti-particle masses. Why should this hold in the framework of sigma models?

Given a particle of mass $m$ and charge $e$, we must show that there is another particle of mass $m$ and charge $-e$. In the context of sigma models, particle masses $m$ are eigenvalues of the sigma model Hamiltonian $H$. $H$ commutes with the world sheet CPT operator $\Theta$, which however anticommutes with conserved charges. Conservation of $\Theta$ thus ensures that for every $H$ eigenstate with eigenvalue $m$ and charge $e$, there is an $H$ eigenstate with eigenvalue $m$ and charge $-e$. Thus, the equality of particle and anti-particle masses in space-time follows directly from the world-sheet CPT theorem. This is an illustration of a general rule by which spacetime phenomena are manifestations of analogous world-sheet phenomena. 
Acknowledgements. I would like to thank M. Hopkins, P. Landweber, S. Ochanine, and R. Stong for discussions.

\section{References}

1. Landweber, P.S., Stong, R.: Circle actions on spin manifolds and characteristic numbers, Rutgers University preprint 1985

2. Atiyah, M.F., Hirzebruch, F.: In: Essays on topology and related subjects, pp. 18-28. Berlin, Heidelberg, New York: Springer 1970

3. Atiyah, M.F., Singer, I.M.: Ann. Math. 87, 484, 586 (1968)

Atiyah, M.F., Segal, G.B.: Ann. Math. 87, 531 (1968)

Atiyah, M.F., Bott, R.: Ann. Math. 87, 456 (1968)

4. Witten, E.: Fermion quantum numbers in Kaluza-Klein theory. In: Shelter Island II: Proceedings of the 1983 Shelter Island conference on quantum field theory and the fundamental problems of physics. Khuri, N. et al. (eds.). Cambridge, MA: MIT Press 1985

5. Borsari, L.: Bordism of semi-free circle actions on spin manifolds. Trans. Am. Math. Soc. (to appear)

6. Ochanine, S.: Sur les genres multiplicatifs définis par des intégrales elliptiques. Topology (to appear)

7. Chudnovsky, D.V., Chudnovsky, G.V.: Elliptic modular functions and elliptic genera. Columbia University preprint (1985)

8. Ochanine, S.: Elliptic genera for $S^{1}$ manifolds. Lecture at conference on elliptic curves and modular forms in algebraic topology. IAS (September 1986)

9. Landweber, P.S., Ravenel, D., Stong, R.: Periodic cohomology theories defined by elliptic curves. Preprint (to appear)

Landweber, P.S.: Elliptic cohomology and modular forms. To appear in the proceedings of the conference on elliptic curves and modular forms in algebraic topology. IAS (September 1986)

10. Hopkins, M., Kuhn, N., Ravenel, D.: Preprint (to appear)

Hopkins, M.: Lecture at the conference on elliptic curves and modular forms in algebraic topology. IAS (September 1986)

11. Dixon, L., Harvey, J.A., Vafa, C., Witten, E.: Strings on orbifolds. Nucl. Phys. B 261, 678 (1985)

12. Schellekens, A., Warner, N.: Anomalies and modular invariance in string theory, Anomaly cancellation and self-dual lattices (MIT preprints 1986). Anomalies, characters and strings (CERN preprint TH 4529/86)

Pilch, K., Schellekens, A., Warner, N.: Preprint, 1986

13. Witten, E.: J. Differ. Geom. 17, 661 (1982), Sect. IV. In: Anomalies, geometry, and topology. Bardeen, W., White, A. (eds.). New York: World Scientific, 1985, pp. 61-99, especially pp. 91.95

14. Atiyah, M.F., Singer, I.M.: Ann. Math. 93, 119 (1971)

15. Zagier, D.: A note on the Landweber-Stong elliptic genus (October 1986)

16. Asorey, M., Mitter, P.K.: Regularized, continuum Yang-Mills process and Feynman-Kac functional integral. Commun. Math. Phys. 80, 43 (1981)

17. Bern, Z., Halpern, M.B., Sadun, L., Taubes, C.: Contınuum regularization of QCD. Phys. Lett. $165 \mathrm{~B}, 151$ (1985)

18. Eichler, M., Zagier, D.: The theory of Jacobi forms. Boston: Birkhäuser 1985

19. Witten, E.: Non-abelian bosonization in two dimensions. Commun. Math. Phys. 92, 455 (1984)

Communicated by A. Jaffe 